Ann. Biol. anim. Bioch. Biophys., I973, 13 (I), I3I-I39.

\title{
UTILISATION MÉTABOLIQUE DES ACIDES GRAS DU TISSU ADIPEUX PAR LA POULE EN PONTE
}

\author{
B. LECLERCQ \\ avec la collaboration technique de Marie-Rose SALICHoN \\ Station de Recherches avicoles \\ Centre de Recherches de Tours, I. N. R. A., \\ 37380 Nouzilly \\ RÉSUMÉ
}

Le tissu adipeux de huit poules pondeuses est marqué par l'acide palmitique-I-14 $\mathrm{C}$. On suit pendant 28 jours l'évolution de l'activité spécifique du carbone du $\mathrm{CO}_{2}$, des acides gras plasmatiques, vitellins et de réserve. La comparaison de ces activités spéci fiques réalisée le $28^{\mathrm{e}}$ jour suggère que $20 \mathrm{p}$. Ioo du carbone des acides gras de l'œuf et $2 \mathrm{p}$. Ioo du carbone du $\mathrm{CO}_{2}$ proviennent des réserves adipeuses. Un parallélisme très étroit existe entre les activités spécifiques des acides gras du plasma et du vitellus. La contribution des réserves pour la vitellogenèse varie beaucoup d'une poule à l'autre et ne peut s'expliquer par l'importance de ces réserves ou par l'intensité de ponte. Seules les consommations élevées d'aliment semblent la réduire.

\section{INTRODUCTION}

Dans une étude précédente ( $\mathrm{L}_{\mathrm{E} C L E R C Q}, \mathrm{I} 972$ ) nous avons montré qu'environ $70 \mathrm{p}$. Ioo du carbone de l'acide palmitique du vitellus provient directement soit du carbone glucidique, soit du carbone de l'acide palmitique contenus dans l'aliment. Nos résultats suggéraient que le carbone provenant des acides gras des réserves adipeuses représente la majeure partie des $30 \mathrm{p}$. Ioo restants. Le présent travail a pour but d'estimer directement cette contribution des réserves corporelles à la fourniture d'acides gras vitellins.

\section{MATÉRIEL ET MÉTHODES}

\section{A. - Protocole expérimental}

Huit poules âgées de zo semaines sont placées en cages individuelles, nourries et abreuvées à volonté. Ces animaux proviennent d'un croisement industriel (Rhode Island Red $\times$ Wyandotte, M 5I9, SELAF, France). La composition de l'aliment figure dans le tableau I. Pendant une période 
de I mois, les animaux sont habitués à des manipulations fréquentes : en particulier, ils effectuent des séjours de 24 heures dans une cage à échange respiratoire. Cette cage, d'une capacité de 80 litres, est suffisamment spacieuse pour que la poule puisse y manifester une activité voisine de celle qu'elle présente habituellement dans sa cage. En outre elle dispose d'eau et d'aliment. $\mathrm{Au}$ cours de cette période destinée à familiariser les poules aux multiples manipulations exigées par l'expérimentation, on détermine la quantité totale de $\mathrm{CO}_{2}$ dégagé en 24 heures.

TABLEAU I

Composition et caractéristiques du régime

\begin{tabular}{|c|c|}
\hline 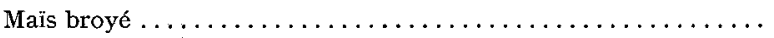 & 60 \\
\hline Tourteau de soja $(50$ p. 100 de MAT) . ................. & 20 \\
\hline 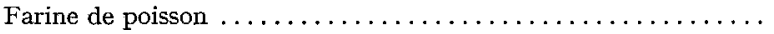 & 2 \\
\hline Farine de luzerne déshydratée $\ldots \ldots \ldots \ldots \ldots \ldots \ldots \ldots$ & 8 \\
\hline 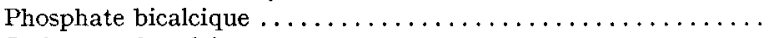 & 1,5 \\
\hline 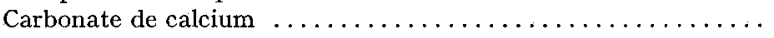 & 8 \\
\hline 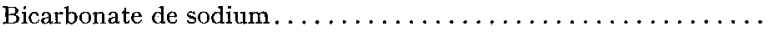 & 0,1 \\
\hline 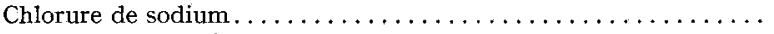 & 0,1 \\
\hline 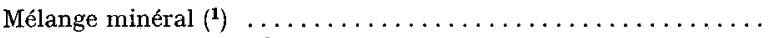 & 0,1 \\
\hline Mélange vitaminique $\left({ }^{2}\right) \ldots$ & 0,2 \\
\hline Teneur en énergie métabolisable $\left({ }^{3}\right)(\mathrm{kcal} / \mathrm{kg})$ & 2920 \\
\hline Taux protidique ${ }^{(4)} \ldots \ldots \ldots \ldots \ldots$ & 18,1 \\
\hline
\end{tabular}

(1) Composition du mélange minéral : protoxyde anhydre de manganèse : $7 \mathrm{~g}$, oxyde de zinc : $5 \mathrm{~g}$, sulfate ferreux $: 7 \mathrm{~g}$, sulfate de cuivre $: 0,5 \mathrm{~g}$, carbonate de cobalt : $0,03 \mathrm{~g}$, iodure de potassium : $0,1 \mathrm{~g}$, carbonate de calcium : $75 \mathrm{~g}$.

(2) Composition du mélange vitaminique : vitamine A : $900000 \mathrm{UI}$, vitamine $\mathrm{D}_{3}: 100000 \mathrm{UI}$, DL-méthionine : $50 \mathrm{~g}$, vitamine $\mathrm{E}: 4 \mathrm{~g}$, choline : $25 \mathrm{~g}, \mathrm{BHT}: 10 \mathrm{~g}$.

(3) Calculé.

(4) Mesuré.

Vient ensuite la période expérimentale qui dure 28 jours. Elle commence par le marquage des réserves adipeuses effectué le premier jour par ingestion en une seule fois d'un acide gras radioactif : après un jeûne de $I 6$ heures, les animaux sont réalimentés et simultanément reçoivent par voie orale $200 \mu \mathrm{Ci}$ d'acide palmitique-I-14C dissous dans l'huile de tournesol. La surconsommation qui accompagne la réalimentation et la lipogenèse qui en résulte laissent espérer un marquage maximum du tissu adipeux. On récolte ensuite régulièrement un certain nombre d'échantillons : prélèvements de sang les $3^{\mathrm{e}}, \mathrm{I}_{4} \mathrm{e}$ et $28^{\mathrm{e}}$ jours par ponction de la veine alaire, prélèvements du $\mathrm{CO}_{2}$ expiré les $\mathrm{I}^{\mathrm{er}}, 2^{\mathrm{e}}, 3^{\mathrm{e}}, 7^{\mathrm{e}}, \mathrm{I}^{\mathrm{e}}, 2 \mathrm{I}^{\mathrm{e}}$ et $28^{\mathrm{e}}$ jours, prélèvement de tissu adipeux abdominal par biopsie les $3^{\mathrm{e}}$ et $28^{\mathrm{e}}$ jours. De plus, du vitellus représentatif de celui synthétisé les $3^{\mathrm{e}}, 7^{\mathrm{e}}$, ${ }^{1} 4^{e}, 2 I^{e}$ et $28^{e}$ jours est recueilli de la façon suivante. La veille et le lendemain du jour considéré, on fait ingérer à la poule un colorant lipophile, soit le rouge écarlate, soit le noir crésol. Les œufs sont récoltés et cuits. On prélève alors par grattage la couche de vitellus située entre les deux couches colorées. Tous les jours on mesure la consommation d'aliment, le nombre et le poids des œufs pondus. Le poids vif des animaux est enregistré en début d'expérience, les $14^{\mathrm{e}}$ et $28^{\mathrm{e}}$ jours. Le poids moyen du jaune d'œuf de chaque poule est également déterminé. Enfin, pour savoir si l'utilisation des réserves varie en fonction de l'apport alimentaire, une légère restriction ( 5 p. roo) est imposée à la moitié des animaux pendant les $\mathrm{I}_{4}$ derniers jours. Le $28^{\mathrm{e}}$ jour les animaux sont sacrifiés par section des veines jugulaires, congelés, broyés et lyophilisés après ablation de la grappe ovarienne et des oufs en cours de formation.

\section{B. - Méthodes d'analyse}

- Gaz carbonique : On détermine d'une part la quantité totale de $\mathrm{CO}_{2}$ dégagé en 24 heures par chaque poule dans des conditions normales de vie et d'autre part l'activité spécifique du gaz carbonique ; ces deux mesures sont réalisées distinctement. Pour la première, faite avant mar- 
quage du tissu adipeux, chaque poule est placée pendant 24 heures dans la cage à métabolisme. Cette cage est balayée par un courant d'air débarrassé de $\mathrm{CO}_{2}$ par barbotage dans la potasse $5 \mathrm{~N}$ et d'eau par passage sur de l'actigel. Le débit est réglé à zoo litres par heure. A la sortie de la cage, l'air est débarrassé de son eau dans deux filtres, l'un contenant du chlorure de calcium, l'autre du perchlorate de magnésium. L'air traverse ensuite deux tamis moléculaires. Le poids de $\mathrm{CO}_{2}$ est déterminé par le gain de poids des tamis.

Pour la mesure de l'activité spécifique du $\mathrm{CO}_{2}$, la poule effectue un séjour de 2 heures dans la cage. Pendant la première heure, on fait circuler de l'air débarrassé d'eau et de gaz carbonique à un débit de 500 litres par heure, la sortie de la cage étant libre. Pendant la deuxième heure, cette sortie est équipée d'un simple barboteur contenant $20 \mathrm{cc}$ de $\mathrm{KOH}$ I,5 N. La quantité de $\mathrm{CO}_{2}$ retenue est déterminée par double titration aux $\mathrm{pH} 7,9$ et 3,5 avec l'acide sulfurique $\mathrm{o}, \mathrm{I} \mathrm{N}$. La radioactivité est mesurée par scintillation liquide en mélangeant $5 \mathrm{~cm}^{3}$ de la solution de barbotage et $\mathrm{I} 7 \mathrm{~cm}^{3} \mathrm{~d}^{\prime}$ un mélange scintillant (toluène $+\mathrm{PPO}+\mathrm{POPOP}$ ) renfermant $33 \mathrm{p}$. Ioo de Triton X Ioo comme agent d'émulsion.

- Lipides : Les lipides du vitellus sont extraits par le mélange chloroforme-méthanol (2-I, v-v), ceux du tissu adipeux et du plasma par le mélange de Dole (isopropanol-heptane$\mathrm{H}_{2} \mathrm{SO}_{4} ; 4$ O-IO-I). Les lipides du tissu adipeux sont directement purifiés par chromatographie préparative sur couche mince de silicagel ; seuls sont prélevés les triglycérides. Les acides gras des autres lipides (plasma et vitellus) sont méthylés. On recueille les esters méthyliques après chromatographie sur couche mince. On mesure enfin la radioactivité par scintillation liquide dans le toluène additionné de PPO et POPOP.

La quantité de carbone des acides gras des triglycérides est obtenue en multipliant leur poids par 0,724 et celui des esters méthyliques par $0,73^{2}$.

\section{RÉSULTATS}

Nous présentons dans la figure I l'évolution de l'activité spécifique du carbone du $\mathrm{CO}_{2}$, des acides gras plasmatiques, vitellins et de réserves au cours des 28 jours d'expérience. Celle du gaz carbonique décroît très rapidement et varie peu entre le

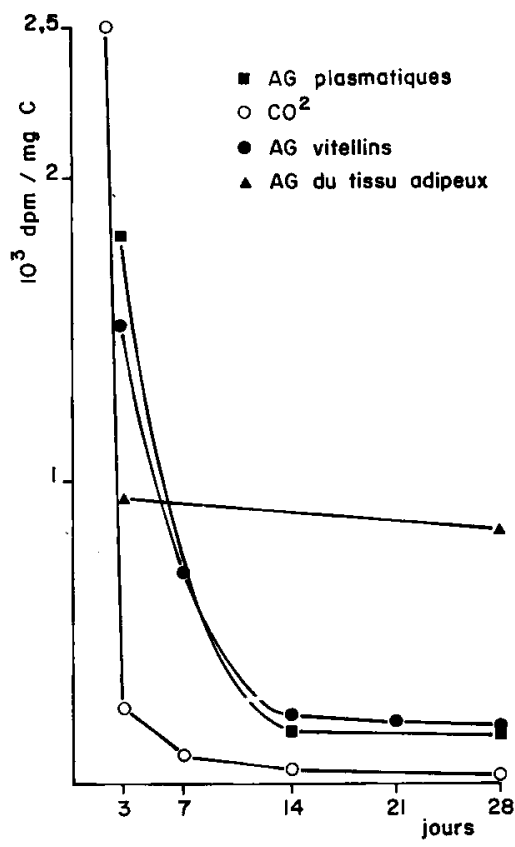

Fì. I. - Evolution de l'activité spécifique du carbone du $\mathrm{CO}_{2}$ et des acides gras vitellins, plasmatiques et adipeux au cours de l'experience 
$7^{\mathrm{e}}$ et le $28^{\mathrm{e}}$ jour. Une décroissance semblable, quoique moins accentuée, est constatée pour les acides gras du plasma et du vitellus ; le parallélisme entre ces deux courbes est par ailleurs remarquable. Entre le $\mathrm{I} 4^{\mathrm{e}}$ et $\mathrm{le} 28^{\mathrm{e}}$ jour, l'évolution des activités spécifiques de ces trois compartiments est très faible. Pendant le même temps, la décroissance de l'activité spécifique du carbone des acides gras de réserves est lente puisqu'elle ne diminue que de ro p. roo en 25 jours.

\section{TABLEAU 2}

État physiologique des poules et activité spécifique du carbone du $\mathrm{CO}_{2}$, des acides gras vitellins, plasmatiques et de réserves au $28 \mathrm{e}$ jour d'expérience
a) Poids moyen des poules ( $g$ )
$1935 \pm 66$
a) Poids d'aliment consommé par 24 heures $(\mathrm{g}) \ldots \ldots$
$102,7 \pm 2,2$
b) Lipides totaux de la carcasse $(g) \ldots \ldots \ldots \ldots \ldots$
$408,5 \pm 36,3$
c) $\mathrm{CO}_{2}$ exporté par 24 heures (g)
$55,4 \pm 2,4$
d) Carbone des acides gras du vitellus exporté par 24 heures (mg) . . . . . . . . . . . . . . .
$2709 \pm 132$
e) Activité spécifique du carbone des acides gras du tissu adipeux $(\mathrm{dpm} / \mathrm{mg}) \ldots \ldots \ldots \ldots \ldots \ldots \ldots$
$838,7 \pm 105,4$
f) Activité spécifique đu carbone des acides gras du vitellus $(\mathrm{dpm} / \mathrm{mg}) \ldots \ldots \ldots \ldots \ldots \ldots \ldots \ldots \ldots$
$174,1 \pm 32,3$
g) Activité spécifique du carbone des acides gras du plasma $(\mathrm{dpm} / \mathrm{mg}) \ldots \ldots \ldots \ldots \ldots \ldots \ldots \ldots$
$156,3 \pm 27,9$
h) Activité spécifique du carbone du $\mathrm{CO}_{2}(\mathrm{dpm} / \mathrm{mg})$..
$16,66 \pm 2,08$
i) Valeur du rapport $f / e \times 100 \ldots \ldots \ldots \ldots \ldots$
$20,57 \pm 2,49$
j) Valeur du rapport $g / e \times 100 \ldots \ldots \ldots \ldots \ldots$
$18,53 \pm 2,07$
k) Valeur du rapport $h / e \times 100 \ldots \ldots \ldots \ldots \ldots$
$2,23 \pm 0,46$
l) Carbone des acides gras vitellins provenant du tissu adipeux par 24 heures $(\mathrm{mg}) \ldots \ldots \ldots \ldots \ldots \ldots$
m) Carbone du $\mathrm{CO}_{2}$ provenant du tissu adipeux par 24 heures $(\mathrm{mg}) \ldots \ldots \ldots \ldots \ldots \ldots \ldots \ldots \ldots \ldots \ldots \ldots \ldots \ldots$. par 24 heures $(\mathrm{mg}) \ldots \ldots \ldots \ldots \ldots \ldots \ldots \ldots$
$568,8 \pm 74,2$
$349,1 \pm 90,8$
$1268 \pm 196$

Dans le tableau 2 nous rapportons différentes mesures effectuées le 28 e jour d'expérience. On peut remarquer que l'activité spécifique du carbone des acides gras vitellins est égale à un peu plus de $20 \mathrm{p}$. roo de celle du tissu adipeux, celle des acides gras plasmatiques est également proche de $20 \mathrm{p}$. Ioo. Au contraire, celle du carbone du $\mathrm{CO}_{2}$ n'est que de 2,23 p. Ioo. En multipliant le rapport

$$
\frac{\text { activité spécifique } \mathrm{du} \text { carbone des acides gras vitellins }}{\text { activité spécifique du carbone des acides gras des réserves }}
$$

par la masse de carbone exportée en moyenne chaque jour dans le vitellus et le rapport

$$
\text { activité spécifique du carbone du } \mathrm{CO}_{2}
$$

activité spécifique du carbone des réserves 
par la masse du carbone exportée en 24 heures sous forme de $\mathrm{CO}_{2}$, on peut estimer la contribution journalière des réserves adipeuses à la formation du vitellus et à celle du $\mathrm{CO}_{2}$. En divisant ces masses de carbone provenant du tissu adipeux par le facteur 0,724 on peut évaluer la quantité d'acides gras puisés chaque jour par lipolyse du tissu gras. Ces résultats figurent dans le tableau 2 ; nous discutons plus loin le bien fondé de ce calcul. On peut cependant constater dès à présent que, d'après ce calcul, la majeure partie du carbone puisé dans le tissu adipeux est destiné à la formation des acides gras de l'œuf.

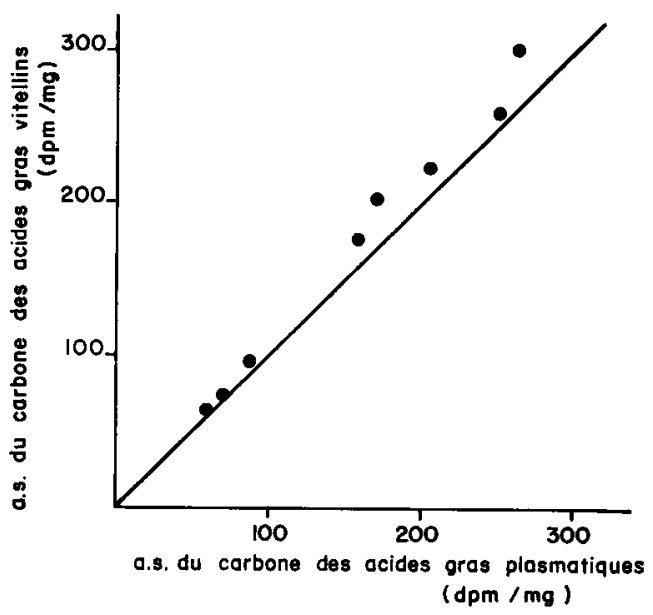

FIG. 2. - Relation entre l'activité spécifique du carbone des acides gras plasmatiques et vitellins

La figure 2 permet de comparer les activités spécifiques du carbone des acides gras vitellins et plasmatiques. On peut constater l'étroite corrélation qui existe entre ces deux mesures.

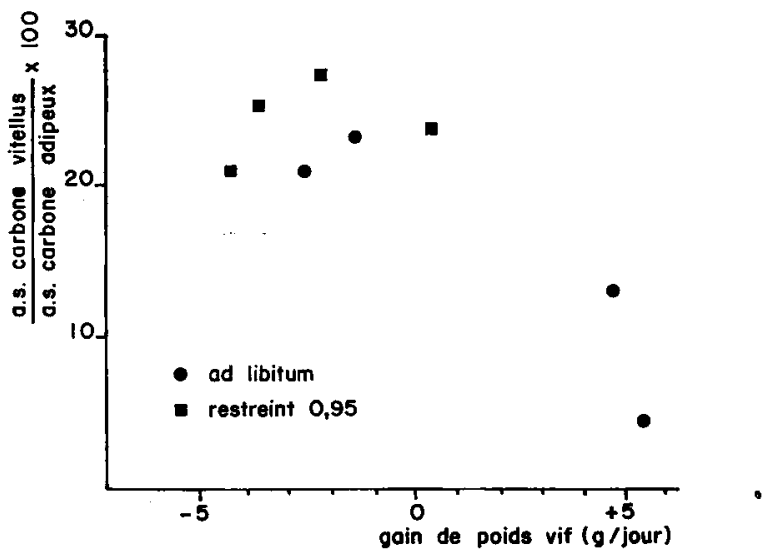

FIG. 3. - Relation entre le gain de poids vif journalier et la valeur du rapport $\frac{A S \text { du carbone vitellin }}{A S \text { du carbone adipeux }}$ 
Dans la figure 3 nous tentons de relier le rapport :

$$
\frac{\text { activité spécifique du carbone des acides gras vitellins }}{\text { activité spécifique du carbone des acides gras de réserves }}
$$

à la variation de poids vif journalier. Cette figure montre d'une part la variabilité du rapport des activités spécifiques selon les individus, d'autre part que les deux animaux mobilisant le moins leurs réserves sont ceux qui présentent le gain de poids le plus important. En dehors de ces deux cas, la liaison entre ces deux critères n'est pas étroite.

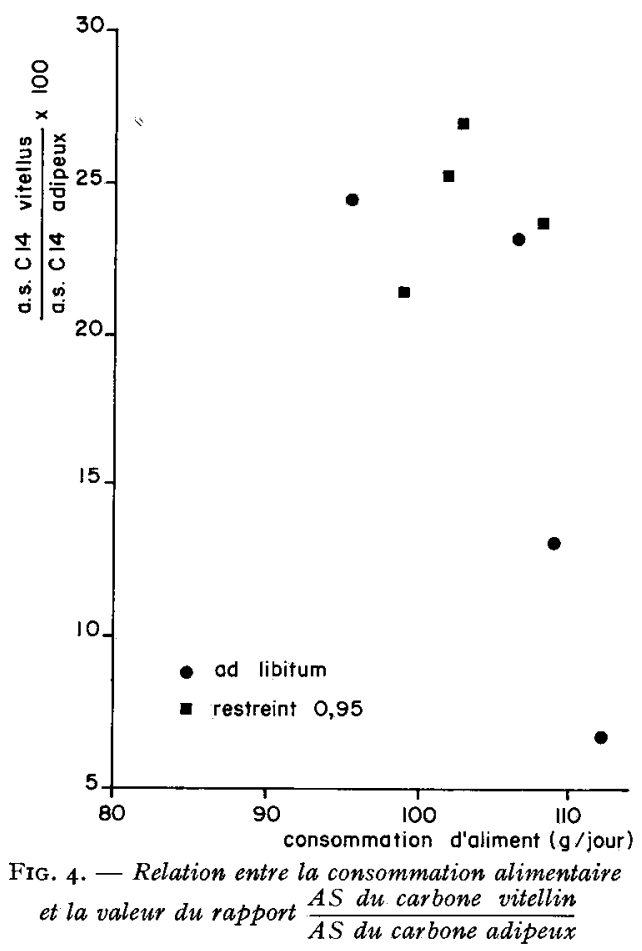

Nous tentons également de relier le rapport des activités spécifiques à la consommation journalière d'aliment grâce à la figure 4. On peut constater, ici encore, qu'il n'existe pas de liaison significative, si ce n'est que les deux poules présentant le rapport d'activités spécifiques le plus faible sont celles qui consomment le plus d'aliment.

\section{DISCUSSION}

L'évolution des activités spécifiques représentées dans la figure I nous suggère qu'entre le début de l'expérience et le I4 ${ }^{\mathrm{e}}$ jour la décroissance des activités spécifiques enregistrées dans le $\mathrm{CO}_{2}$, le plasma et le vitellus correspond au renouvellement des pools très actifs de l'animal (foie et sang) marqués par le carbone radioactif d'ori- 
gine alimentaire. Au-delà de cette période et d'après ce que 1'on sait de la vitesse de renouvellement de ces pools (ENTENMAN et al., I946 ; HAVEL, et al., I962 ; BAKER et al., I964; OETTE et al., I97I), on peut penser que la seule source notable de carbone radioactif est le tissu adipeux. Comme, par ailleurs, son activité spécifique ne décroît que très lentement, on est dans des conditions très proches de l'équilibre isotopique permettant d'évaluer la contribution du pool du carbone des acides gras de réserves pour la synthèse du vitellus et la formation du gaz carbonique. Il faut aussi admettre qu'il n'existe pas dans le tissu adipeux de la poule, pour les acides gras autres que l'acide linoléique, plusieurs pools dont l'activité et la taille varient selon la nature de ces acides gras et selon l'âge de leur dépôt in situ. Cela paraît vraisemblable puisqu'il en est ainsi chez le Rat (MENDENHALI, I972). Ces deux hypothèses étant admises, les calculs présentés en fin du tableau 2 paraissent justifiés.

On peut donc estimer que près de 20 p. Ioo des acides gras du vitellus proviennent du tissu adipeux. Si l'on rapproche ce chiffre des $70 \mathrm{p}$. Ioo trouvés dans un essai précédent (LECLERCQ, I972) et qui correspond à la contribution du carbone alimentaire d'origine glucidique ou lipidique, ou aboutit à un bilan proche de $90 \mathrm{p}$. roo. Le reste relève soit des conditions expérimentales différentes, soit de la contribution d'autres voies métaboliques telles que : carbone provenant de la $\beta$-oxydation des acides gras alimentaires, carbone d'origine protidique... Cette estimation de l'origine des acides gras vitellins est à rapprocher d'autres travaux. Ainsi, selon MENDENHALL (I972), 24,5 p. IOo des triglycérides du foie du Rat proviennent du tissu adipeux. La part du carbone du $\mathrm{CO}_{2}$ provenant du tissu adipeux est au contraire remarquablement faible. Si l'on tient compte de la masse de carbone exporté sous forme de $\mathrm{CO}_{2}$ on sous forme d'acides gras vitellins, on constate, comme le montre le tableau 2 que les acides gras provenant du tissu adipeux servent beaucoup plus à la fabrication des lipides du vitellus qu'à la couverture des besoins énergétiques. Cette destinée privilégiée des acides gras des réserves s'oppose à celle des acides gras alimentaires dont la plus grande part sert à la couverture des dépenses énergétiques de 1'anima1 (LECLERCQ, I972). L'explication pourrait être recherchée dans la forme de transport de l'acide gras : forme libre s'il provient du tissu adipeux, triglycérides s'il provient de l'aliment.

La contribution des graisses de réserves pour la vitellogenèse est l'objet de variations importantes d'un individu à l'autre. Elles ne peuvent s'expliquer ni par l'importance de ces réserves (lipides de la carcasse), ni par l'intensité de la vitellogenèse. On peut seulement remarquer que cette contribution tend logiquement à être plus faible chez l'animal qui consomme beaucoup d'aliment, bien que cette liaison ne soit pas très étroite pour l'ensemble des animaux. Cause ou conséquence, le gain de poids vif est plus élevé chez les poules qui utilisent peu leurs réserves corporelles pour la synthèse de l'œuf. Apparemment, il existe des contrôles physiologiques décidant de la part respective des apports alimentaires (appétit des animaux) et des réserves (lipolyse) pour la synthèse des acides gras de 1'œuf.

Enfin il faut remarquer une fois de plus le parallélisme remarquable entre les lipides plasmatiques et vitellins, comme le révèlent les figures I et 2 . Ce parallélisme, de même que les études biochimiques effectuées sur les lipoprotéines de l'œuf et du sang (GORNHALI, et al., I97I ; HIL,YARD et al., I972), suggèrent que l'ovaire n'a pas d'activité importante au cours de la vitellogenèse. Ses activités de synthèse et de filtration doivent être réduites, tout au moins en ce qui concerne les lipides. 


\section{CONCLUSION}

Les résultats que nous venons de présenter permettent d'estimer la quantité journalière d'acides gras mobilisés à $I, 3 \mathrm{~g}$. Dans la mesure où nos deux expériences peuvent être rapprochées, il apparaît bien que le dépôt excède la mobilisation, mais que la différence n'est pas aussi importante que nous l'avions suggéré. Néanmoins, même si la mobilisation des réserves atteint ce niveau, elle ne concerne que $0,3 \mathrm{p}$. Ioo des réserves par 24 heures.

Nos résultats montrent que le dépôt comme la mobilisation des réserves varient beaucoup d'une poule à l'autre. Nous ne savons malheureusement pas quelle liaison existe pour un même animal entre ces deux phénomènes dont le bilan détermine l'état d'engraissement. Comme on constate par ailleurs que celui-ci varie beaucoup selon l'alimentation, l'âge, la souche et même les individus, il nous paraît intéressant de poursuivre l'étude des facteurs nutritionnels et génétiques qui influencent la lipolyse et la lipogenèse. Nous tenterons à cette occasion de mesurer les deux phénomènes sur chaque animal.

Reçu pour publication en octobre 1972.

\section{METABOLIC UTILIZATION OF THE FATTY ACIDS OF ADIPOSE TISSUE BY THE I,AYING HEN}

The tissue of eight hens 30 weeks old was marked with palmitic acid-I-14 $\mathrm{C}$ by mouth in oily solution on the first day of the experiment. Changes in specific activity of the carbon of $\mathrm{CO}_{2}$, of fatty acids of plasma, egg yolk and adipose tissue were followed for 28 days (fig. I). Table 2 shows the balance of these specific activities on the 28 th day of the experiment. In figure 2 we present the correlation between the specific activities in fatty acids of plama and yolk. The relation between daily liveweight gain and the ratio

$$
\underline{\text { specific activity of carbon of fatty acids in yolk }}
$$

are the subject of table 3 . The relations of that same ratio with feed intake is presented in table 4 .

From these results we conclude that 20 per cent of the yolk fatty acids come from adipose tissue and that only 2.2 per cent of the carbon of $\mathrm{CO}_{2}$ is from carbon of adipose tissue. Taking in to account the amounts of $\mathrm{CO}_{2}$ and fatty acids produced in 24 hours, it may be calculated that the hen draws from its reserves on average $568.8 \mathrm{mg}$ carbon to form fatty acids of the yolk and $349 \mathrm{mg}$ carbon for production of $\mathrm{CO}_{2}$. In total it can be estimated that in our conditions I $270 \mathrm{mg}$ fatty acids are drawn from adipose tissue in $24 \mathrm{~h}$, which represents a turnover of 0.3 per cent per 24 hours.

The mobilization of reserves varies widely from hen to hen. It cannot be related to the amount of the reserve or the rate of yolk formation. There is a weak relation between this mobilization and feed intake, giving rise equally to a weak negative correlation between weight gain and rate of lipolysis.

We propose to study simultaneously in the same animals the rates of lipogenesis and lipolysis, and to establish the importance of nutritional and genetic factors. 


\section{RÉFÉRENCES BIBLIOGRAPHIQUES}

Baker N., Schotz M. C., I964. Use of multicompartmental models to measure rates of triglycerides metabolism in rats. J. Lipid Res., 5, I88-197.

Entenman C., Chaikoff I. L., Zilversmith D. B., I946. Removal of plasma phopholipids as a a function of the liver : the effect of exclusion of the liver on the turn-over rate of plasma phospholipids as measured with radioactive phosphorus. J. Biol. Chem., 166, I5-23.

Gornhall D. A., Delahunty D. A., Kuksis A., I97I. Immunochemical relationship among plasma and egg yolk lipoproteins of the laying hen. Bioch. J., 125, $97 \mathrm{p}$.

Havel R. J., Felts J. M., Vanduyne C. M., 1962. Formentation and fate of endogenous triglycerides in blood plasma of rabbits. J. Lipid Res., 3, 297-308.

Hillyard L. A., White H. M., Pangburn S. A., I972. Characterization of apolipoproteins in chicken serum and egg yolk. Biochemistry, 11, $5 \mathrm{I} \mathrm{x}-5 \mathrm{r} 8$.

Leclerco B., I972. Étude de la biosynthèse et de l'utilisation des acides gras par la poule pondeuse. Ann. Biol. anim. Bioch. Biophys., 12, 44I-452.

Mendenhall C. L., I972. Origin of hepatic triglycerides fatty acids : quantitative estimation of the relative contributions of linoleic acid by diet and adipose tissue in normal and ethanol-fed rats. $J$. Lipid Res., 18, I77-I83.

Oette K., Frese W., Phlippen R., I97I. Studies on the uptake of chylomicron fatty acids into the human liver and the turn-over of dietary fatty acids within the endogenous fatty acid cycle. Zeitsch. ges Exp. Med., 154, 208-223. 